\title{
Strategien für den Gesundheitsmarkt Kongress Klinikmarketing 2011
}

Eine Erfolgsgeschichte: Dynamisch steigende Beteiligung in den letzten Jahren, allein von 2010 auf 2011 einen Zuwachs von $60 \%$ und damit de facto einen Anstieg von 350 auf 561 Teilnehmerinnen und Teilnehmer verzeichnet der Kongress Klinikmarketing, der vom 21. bis 25 . November 2011 in Köln stattfand.

Stefan Rotthaus, Geschäftsführer des Veranstalters rotthaus.com, erklärt sich dies damit, dass immer mehr Kliniken nicht nur erkennen, dass Marketing für sie erfolgsrelevant wird, sondern dafür auch aktiv Strategien entwickeln wollen. Seit 6 Jahren ist der Kongress in Köln die Plattform für professionelles Klinikmarketing in Deutschland, Österreich und der Schweiz. Den wachsenden Anforderungen und den steigenden Teilnehmerzahlen trug rotthaus. com 2011 mit einer durchdachten komplett neu gestaltenen Kongressstruktur Rechnung, die den konstruktiven Erfahrungsaustausch mit den mehr als 120 Expertinnen und Experten, wie auch der Teilnehmer und Teilnehmerinnen untereinander noch besser möglich machte. Kern der Veränderungen ist ein neuer Blick auf das Klinikmarketing mit praxisnahen Klinikbeispielen und einem systematischen Aufbau mit 8 ganztägigen Plattformen, die klar strukturiert alle Themenfelder des Klinikmarketings abdecken: Pressearbeit, Personalmarketung und Recruiting, Nutzung von
Web, Film und Social Media, Zuweisermarketing und Vertrieb, Fallzahlen- und Erlössteigerung bis hin zu Erfolgswerkzeugen für leitende Ärzte und der oft sträflich vernachlässigten Patienten- und Mitarbeiterkommunikation, nach Rotthaus einer der entscheidenden Faktoren eines künftigen Klinikerfolgs.

\section{Gesellschaftlicher Höhepunkt:}

die Verleihung der KlinikAwards

Die Jury des KlinikAward 2011 hatte aus über 100 Einsendungen und Vorschlägen von Akut- und Rehakliniken aus Deutschland, Österreich und der Schweiz 34 Kliniken für den KlinikAward nominiert, auch als „Oskar des Klinikmanagements“ bezeichnet. Das Fachpublikum des Kongresses entschied in 5 der 9 Award-Kategorien. Nur der/die „Manager/in des Jahres" und die Kategorie "Bestes Klinikmarketing" wurden allein von der Jury aus einer Vielzahl von Vorschlägen aus der Branche auserwählt und erst zur Verleihung bekannt gegeben. Die KlinikAwards wurden im Rahmen des Gala-Abends am 22. November 2011 vergeben - und zwar für

- „Bestes Klinikfundraising“ an die Vestische Kinder- und Jugendklinik in Datteln

- „Bestes Rehamarketing“ an die Luise von Marillac-Klinik in Bad Überkingen
Kongress Klinikmarketing 2012

Bitte vormerken oder besser gleich anmelden: 8. Kongress Klinikmarketing vom 5.bis 9. November 2012 in Köln. Lernen und diskutieren mit den Profis und Kollegen/Kolleginnen und wieder mit vielen praxisnahen Beispielen, quergedacht - visionär - branchenübergreifend und mit für Sie maßgeschneiderten Strategien, Ideen und Lösungen. Mehr Informationen auf www.kongress-klinikmarketing.org

- „Bestes Zuweisermarketing“ an das Krankenhaus der Barmherzigen Schwestern in Linz

- „Bestes Personalmarketing“ an das St.Franziskus-Hospital in Münster

- Manager/in des Jahres“ an Irman Gürkan vom Universitätsklinikum in Heidelberg

- „Bestes Geburtshilfemarketing“ an das Evangelische Diakoniekrankenhaus in Freiburg

- „Bester Kinikfilm“ an das Katholisches Marienkrankenhaus in Hamburg

- „Bestes Klinikmarketing“ an die Privatklinikgruppe Hirslanden in Zürich

- „Beste Marketinginnovation“ an das Krankenhaus Barmherzige Brüder in Regensburg

Mit dem KlinikAward werden hervorragende Leistungen im Bereich Klinikmarketing des deutschsprachigen Raums ausgezeichnet und ein Anreiz für mehr Transparenz und Wettbewerb im Klinikmarkt geschaffen.

Günther Buck, Weilheim an der Teck

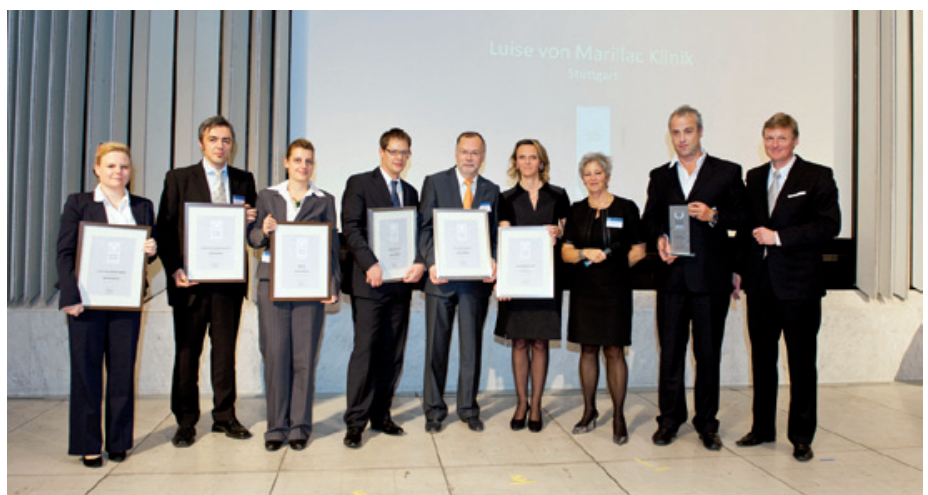

Abb.1 Von Links: Nominiert: Barbara Jung, Katholisches Klinikum Duisburg, Leitung Presse und Marketing; Nominiert: Ronald Poljak, Klinikum der Johann Wolfgang Goethe Universität, EKE Manager; Nominiert: Anja Lamm, MediClin, Personalreferentin; 3. Preis: Björn Kasper, Kliniken Essen-Mitte, Leiter Marketing \& Kommunikation; 2. Preis: Dr. Richard Röhrig, Alice Hospital Darmstadt, Kfm. Geschäftsführer; 1. Preis: Monika Röther, Geschäftsführerin, Luise von Marillac Klinik; Moderatorin: Dr. Elizabeth Harrison, Klinikum München, Geschäftsführerin; Sponsor: Tobias Bartel, KiK-TV Klinik Info Kanal, Geschäftsführer; Initiator des Kongress Klinikmarketing: Stephan Rotthaus, rotthaus.com, Geschäftsführender Gesellschafter

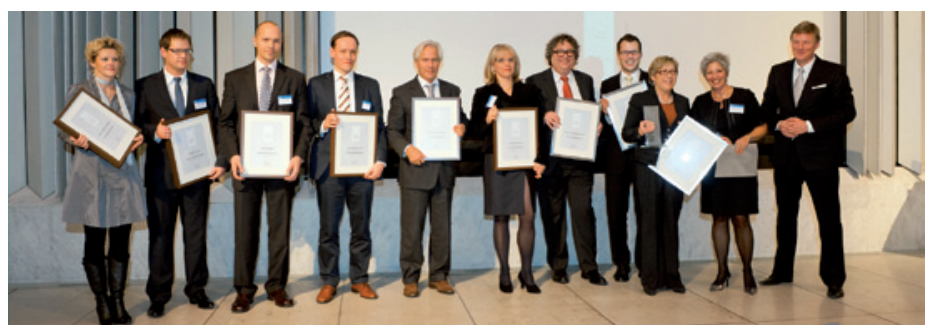

Abb. 2 Von Links: Nominiert: Kerstin Borys, Asklepios Klinikum Uckermark, Stationsleitung und Präventionsbeauftragte der Klinik für Kinder- und Jugendmedizin; Nominiert: Björn Kasper, Kliniken Essen-Mitte, Leiter Marketing \& Kommunikation; Nominiert: Dieter Schleier, Ortenau Klinik, Leiter Unternehmenskommunikation und Marketing; Nominiert: Alexander Friebel, Parkkrankenhaus Leipzig, Leiter Unternehmenskommunikation; Nominiert: Marc Pfirter, Regionales Pflegezentrum Baden, Direktor; Nominiert: Christina Jacke, St. Augustinus Kliniken, Marketingbeauftragte; 3. Preis: Holger Steudemann, Vivantes Netzwerk für Gesundheit, Leiter Interne Kommunikation und Marketing; 2. Preis: Claus Hager, Krankenhaus der Barmherzigen Schwestern Linz, Leitung PR und Marketing; 1. Preis: Sabine Beiser, Krankenhaus Barmherzige Brüder Regensburg, Geschäftsführerin; Dr. Elizabeth Harrison, Klinikum München, Geschäftsführerin; Initiator des Kongress Klinikmarketing: Stephan Rotthaus, rotthaus.com, Geschäftsführender Gesellschafter 